\title{
The Observation of Laser-Induced Transient Species in a Molecular Charge-Transfer System Using X-Ray Photoelectron Spectroscopy $†$
}

\author{
Nicklas Johansson, ${ }^{a+*}$ Thomas Kugler, $^{a}$ Lennart Eberson ${ }^{b}$ and William R. Salaneck ${ }^{a}$ \\ ${ }^{a}$ Department of Physics, IFM, Linköping University, S-581 83 Linköping, Sweden and ${ }^{b}$ Chemical Center, \\ Lund University, PO Box 124, S-221 00 Lund, Sweden
}

\section{Dedicated to Professor Lennart Eberson on the occasion of his 65th birthday}

\begin{abstract}
Johansson, N., Kugler, T., Eberson, L. and Salaneck, W. R., 1998. The Observation of Laser-Induced Transient Species in a Molecular Charge-Transfer System Using X-Ray Photoelectron Spectroscopy. - Acta Chem. Scand. 52: 275-279. (C) Acta Chemica Scandinavica 1998.

The photochemistry of naphthalene-tetranitromethane has been studied at $90 \mathrm{~K}$ by X-ray photoelectron spectroscopy in nanometre thick films, deposited on substrates at low temperatures, with particular emphasis on developing a method for the detection and study of relatively long-lived transient species.
\end{abstract}

Tetranitromethane (TNM) and aromatic donors (ArH) are known to form weak charge-transfer complexes. In a diethyl ether glass at $77 \mathrm{~K}$, upon irradiation with light of $\lambda=405$ or $436 \mathrm{~nm}$, such complexes are known to undergo internal electron transfer to yield a triad of the aromatic radical cation $\left(\mathrm{ArH}^{+}\right)$, trinitromethanide ion and $\mathrm{NO}_{2}$ [eqn. (1)]. These results have been deduced from UV

$\mathrm{ArH} \cdots \mathrm{C}\left(\mathrm{NO}_{2}\right)_{4} \stackrel{h v}{\longrightarrow}\left(\mathrm{ArH}^{+}\right) \mathrm{NO}_{2}\left(\mathrm{NO}_{2}\right)_{3} \mathrm{C}^{-}$

and EPR spectroscopic studies. ${ }^{1}$ The disappearance of the radical cation under the influence of light was suggested to involve electron transfer between the trinitromethanide ion and $\mathrm{ArH}^{+}$. The presence of the radical anion of tetranitromethane was not detected, since it is cleaved to the trinitromethanide ion and $\mathrm{NO}_{2}$, either concurrent with photoelectron transfer or very rapidly thereafter. Later, extensive studies in fluid solution (mostly dichloromethane) established ${ }^{2}$ that the lifetime $\tau_{1 / 2}$ of $\mathrm{C}\left(\mathrm{NO}_{2}\right)_{4}{ }^{\cdot-}$ was $<3 \mathrm{ps}$ at room temperature, subsequently ${ }^{3}$ revised to $\tau_{1 / 2}<10 \mathrm{ps}$. The nature of the $\left(\mathrm{ArH}^{+}\right)-\left(\mathrm{NO}_{2}\right)_{3} \mathrm{C}^{-}$reaction was shown to be bond formation, producing a trinitromethylcyclohexadienyltype neutral radical, capable of very fast reaction with

\footnotetext{
* To whom correspondence should be addressed.

† The subject matter addressed in this paper has been one of Lennart Eberson's favorite subjects over recent years. We feel fortunate to have been able to produce the, so far preliminary, results discussed below, in close cooperation with him. We hope that he will enjoy reading this.
}

$\mathrm{NO}_{2}$ to form stable, often isolable, nitro- or nitritotrinitromethyl adducts to aromatic ring systems, ${ }^{4,5}$ as indicated in eqn. (2). In the absence of $\mathrm{NO}_{2}$, electron transfer takes place. ${ }^{6}$

$$
\begin{aligned}
& \mathrm{ArH}^{+}+\left(\mathrm{NO}_{2}\right)_{3} \mathrm{C}^{-} \stackrel{(1)}{\longrightarrow} \operatorname{Ar}(\mathrm{H}) \mathrm{C}\left(\mathrm{NO}_{2}\right)_{3} \cdot \stackrel{\mathrm{NO}_{2}}{\longrightarrow} \\
& \operatorname{Ar}(\mathrm{H})\left(\mathrm{NO}_{2}\right) \mathrm{C}\left(\mathrm{NO}_{2}\right)_{3}+\operatorname{Ar}(\mathrm{H})(\mathrm{ONO}) \mathrm{C}\left(\mathrm{NO}_{2}\right)_{3}
\end{aligned}
$$

In view of the above results, it was of interest to see whether the photochemistry of $\mathrm{ArH}-\mathrm{C}\left(\mathrm{NO}_{2}\right)_{4}$ combinations could be studied by $\mathrm{X}$-ray photoelectron spectroscopy $^{7}$ in nanometre thick films, deposited on substrates at low temperatures, with particular emphasis on detecting transient species which might correspond to $\mathrm{ArH}^{+}$or species resulting from further transformations of the triad in eqn. (1). Below follows a preliminary account of the application of this method to the naphthalene-tetranitromethane reaction, chosen because of the extensive studies devoted to naphthalene and its methyl derivatives. ${ }^{4}$ The reaction is summarized in Fig. 1

\section{Experimental}

X-Ray photoelectron spectroscopy, or XPS, often known as ESCA, was carried out in a UHV spectrometer of our own design and construction. The system consists of sample introduction, sample preparation and sample analysis chambers. Pumped by a special combination of cryo, ion and turbo pumps, the vacuum in the preparation and analysis chambers is $p<10^{-10}$ Torr, enabling work at very low temperatures without significant prob- 
I<smiles>c1ccc2ccccc2c1</smiles>

II

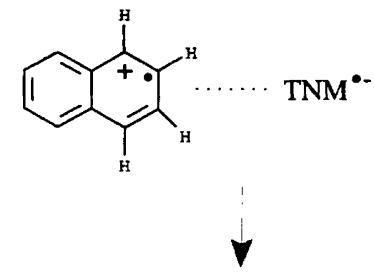

III<smiles>O=C(C=Cc1ccc2ccccc2c1)O[Na]</smiles>
IV<smiles>CC1=CC=Cc2ccccc2C1(C)C</smiles>
V

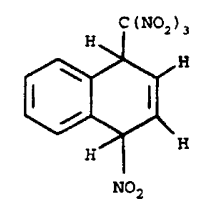

Fig. 1. A summary of the reaction path followed by naphthalene and tetranitromethane, or TNM, from Refs. 4 and 5. Note that there exists another isomer for the products in step V.

ability that surface contamination will occur due to condensed background gases in the spectrometer. The $\mathrm{X}$-ray photons are derived from a non-monochromatized $\mathrm{Mg} K \alpha$ source, and the electron energy analyzer is biased for a resolution such that the $\operatorname{Au}\left(4 \mathrm{f}_{7 / 2}\right)$ line would be recorded with a FWHM of $0.9 \mathrm{eV}$. The scheme used for the preparation of the samples for this set of experiments is shown schematically in Fig. 2. At each stage, despite the excellent vacuum in the system, the substrate temperatures were kept as high as possible in order to minimize further chances of adsorbed surface contamination. To prepare a given sample, a substrate, consisting of a $3000 \AA$ layer of gold on an optically flat silicon (110) wafer, was cleaned in situ by $\mathrm{Ne}^{+}$ion etching to remove hydrocarbon contamination. Then a thin film of naphthalene was deposited, at a partial pressure of $1 \times 10^{-8}$ Torr, on the gold surface, held at $150 \mathrm{~K}$. The thickness of the naphthalene film was monitored by observing the decreasing intensity of the $\mathrm{Au}\left(4 \mathrm{f}_{7 / 2}\right)$ line from the substrate. The estimated thickness of the naphthalene layers ranged from 20 to $50 \AA$, of which $\approx 20 \AA$

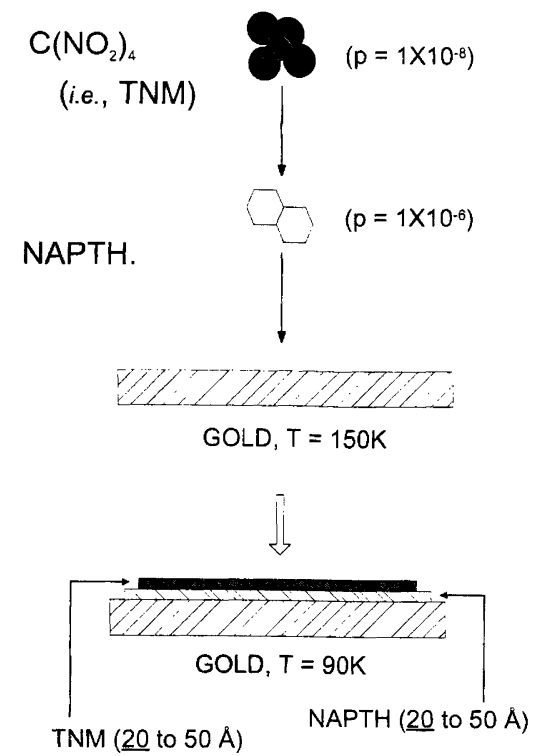

Fig. 2. The sample preparation scheme shown is described in the text.

was typical. Then an overlayer of tetranitromethane was deposited on top of the naphthalene layer, from a partial pressure of $1 \times 10^{-6}$ Torr, with the naphthalene-gold substrate being held at $90 \mathrm{~K}$. The thickness of the tetranitromethane overlayers ranged from 20 to $50 \AA$, of which again $\approx 20 \AA$ was typical. In a typical run, a sample was prepared and run over a course of $4-5 \mathrm{~h}$, during which time no evidence of hydrocarbon contamination was observed. The light exposure was from an unfiltered $\mathrm{Ar}^{+}$laser for which the dominant line is at $488.0 \mathrm{~nm}$. The total intensity was adjusted, by trial and error, to about $0.2 \mathrm{~W} \mathrm{~cm}^{-2}$ over the entire area of a given sample, which was enough that the effects reported below could be observed, but not enough to evaporate off the sample from the substrate in UHV, as higher intensity exposures did. The exposure times were controlled by a mechanical shutter and are given in connection with discussions of the spectra below.

\section{Theoretical methodology}

In order to facilitate the assignment of the experimentally observed XPS spectra, $a b$ initio quantum chemical calculations were performed on some of the suggested products; the triad [see eqn. (1]) was not among the products calculated, since it is a formidable task, in itself, just to obtain the correct geometry for this excited complex. The chemical and electronic structures were calculated using the $3-21 \mathrm{G}$ basis in the GAUSSIAN 94 package. The $\mathrm{C}(1 \mathrm{~s})$ binding energies of carbon atoms in different products are estimated by the differences in electrostatic potential on each atom. Final state effects have not been included, so far, assuming that these are similar for all compounds. It should be emphasized that the purpose of the calculations was to assist in the assignment of the 
XPS peaks and not to calculate the precise chemical shifts. The chemical shifts are either related to the position of the peak in pure naphthalene or the peak in pure TNM.

\section{Results and discussion}

For condensed naphthalene films, only a C (1s) line was observed, near $284 \mathrm{eV}$ binding energy, as expected. The purity of the naphthalene film could be verified by observation of the weak shake-up satellite structure on the high binding energy side of the main $\mathrm{C}(1 \mathrm{~s})$ line, which is unique to naphthalene, compared with possible hydrocarbon contamination. ${ }^{8}$ For the tetranitromethane overlayer, because of the influence of the four electronwithdrawing nitro groups, the $\mathrm{C}(1 \mathrm{~s})$ line is chemically shifted and appears at higher binding energy (near $292 \mathrm{eV}$ ) than that from the naphthalene underlayer, enabling a clear distinction between the two $\mathrm{C}(1 \mathrm{~s})$ lines in the spectra, as shown in Fig 3. Although the N (1s) and $\mathrm{O}(1 \mathrm{~s})$ lines also were recorded, indicating the correct chemical composition expected for the tetranitromethane molecule, these lines are not presented in figures here.

Following the above, a typical sample consisted of about $20(+) \AA$ of tetranitromethane on top of about $20(+) \AA$ of naphthalene, for which the C (1s) spectrum showed the two separated lines in the middle curve of Fig. 3. Two issues should be pointed out here: (1) because only a weak charge-transfer complex (binding energy of the order of $0.1 \mathrm{eV})^{2}$ is expected between tetranitromethane and naphthalene (in the absence of exposure to the laser light), and because of the limited energy resolution of the present equipment (unfiltered $\mathrm{Mg} K \alpha$ radiation), the chemical shifts associated with the initial weak charge-transfer complexing are not observed; and (2) since the elastic mean free path (escape depth) of the photogenerated electrons from the $\mathrm{Au}\left(4 \mathrm{f}_{7 / 2}\right)$ is $\tau \approx 22 \AA,{ }^{9}$ the naphthalene-tetranitromethane interface can be seen through the TNM overlayer [hence the two C (1s) peaks in Fig. 3]. This value of $\tau$ renders the interface visible in the spectra through about $20 \AA$ of tetranitromethane, yet provides the surface sensitivity needed for these studies. Upon increasing the temperature of a sample, without exposure to the laser light, both the tetranitromethane and naphthalene layers evaporate in the UHV system, leaving nothing behind on the Au substrate. This proves that no chemical reactions occurred between the naphthalene and tetranitromethane molecules at the interface in the absence of light. Finally, the possibility that some inter-diffusion of the molecules at the tetranitromethane-naphthalene interface occurred, cannot be excluded. However, from the spectra it is clear that any such diffusion is on a distance scale $(d)$ which is small compared with the respective layer thicknesses, i.e., $d<20 \AA$.

Upon exposure to laser light, photochemistry occurs at the tetranitromethane-naphthalene interface. The maximum exposure level allowed for the present experiments is dictated by the requirement that the tetranitro-

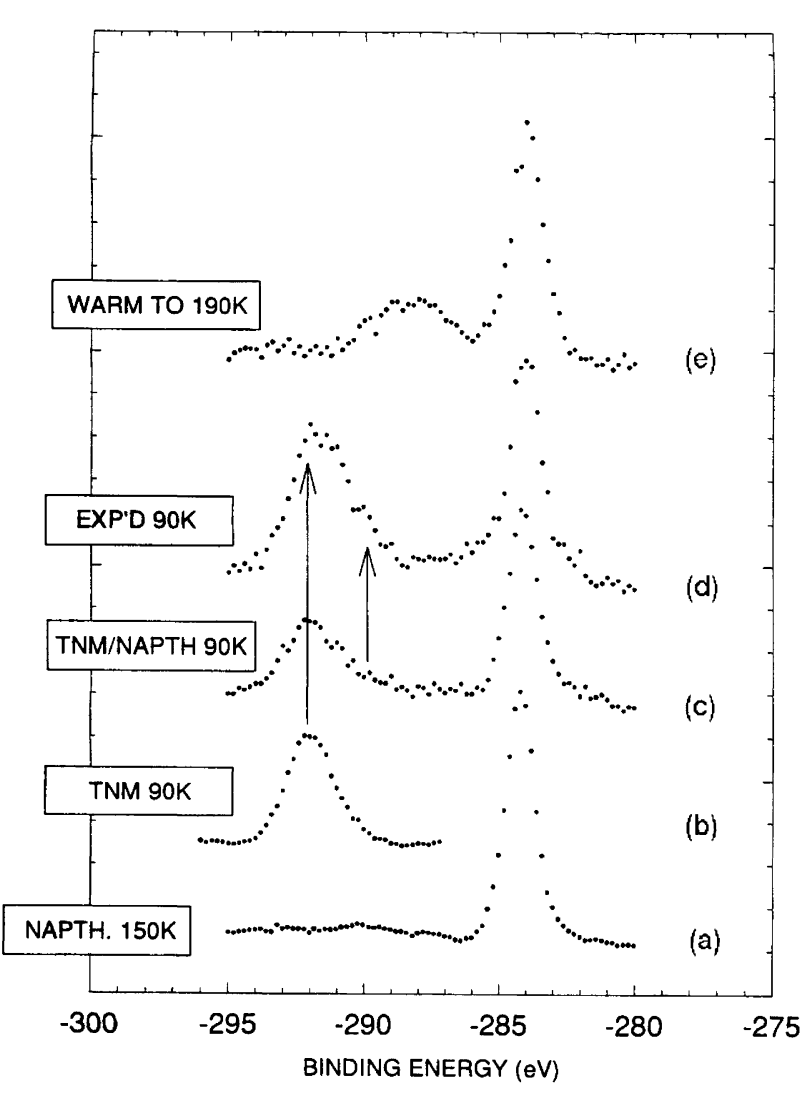

Fig. 3. The $C(1 s)$ spectra of the sample stages for one particular sample run: (a) naphthalene on gold; (b) tetranitromethane, condensed on gold at $90 \mathrm{~K}$ in a separate run; (c) tetranitromethane condensed on naphthalene (on gold); (d) tetranitromethane condensed on naphthalene (on gold) following several exposures of the $\mathrm{Ar}^{+}$ion laser (see the text); and (e) after warming the exposed sample to $190 \mathrm{~K}$.

methane and naphthalene layers do not evaporate upon exposure due to heating of the substrate. At the dictated exposure level, the cross-sections for the events to be discussed below are apparently such that the interface acts as a reservoir for certain photo-induced molecular reactions, a reservoir which is depleted over the course of the measurement time. The rate of the resultant reactions is such that a simple form of time-dependent XPS may be carried out 'by hand'. The C (1s) spectrum can be taken, within a reasonable statistical limit, to capture a picture of a transient state remaining after a 1-min laser exposure at the energy level described above. Upon increasing the temperature of the light exposed sample, simple evaporation of the tetranitromethane and naphthalene layers is not observed. Instead, there remain chemically reacted (transformed) species, with a changed XPS signature, up to much higher temperatures than would otherwise be required to evaporate the unreacted molecular species. The C (1s) spectra at several stages of the experiment are shown in Fig. 3.

Following a 1-min light exposure, in the first scan (requiring with the present equipment $\approx 35 \mathrm{~s}$ ) there appears an obvious shoulder on the high binding energy 
side of the naphthalene $\mathrm{C}$ (1s) peak, a shoulder which is not obvious in the second scan (without additional laser exposure). In order to acquire better statistics on the transient shoulder, the experiment, on each of several individual samples, was repeated as follows: the sample was prepared and appropriate spectra recorded; a single narrow-scan of the naphthalene C (1s) line was recorded; the sample was exposed to $1 \mathrm{~min}$ of $0.2 \mathrm{~W} \mathrm{~cm}^{-2}$ of the unfiltered laser light; a separate naphthalene C (1s) single scan was recorded; the sample was rested in the dark for $5 \mathrm{~min}$; the exposure was repeated; a separate naphthalene $\mathrm{C}$ (1s) scan was recorded; and the process repeated until the transient shoulder no longer appeared, i.e., the interfacial reservoir was depleted. In Fig. 4 is shown a C (1s) spectrum taken following a $1 \mathrm{~min}$ light exposure, as well as a summary of seven such spectra, each acquired separately following additional $1 \mathrm{~min}$ exposures. The summary spectrum is compared with the single scan in the figure.

The effect observed may be summarized as follows: a light-induced species exhibiting a $\mathrm{C}(1 \mathrm{~s})$ peak with higher binding energy than that of naphthalene appears in the region of the interface. which disappears within a period of the order of the scanning time. The transient species contains some positive charge compared with naphthalene. From the chemical shift of the shoulder peak, the small deconvoluted peak of Fig. 4, using generalized CNDO charges vs. measured chemical shift data, ${ }^{7}$ a lowest order approximation of the amount of transferred charge is $\approx 0.14 \mathrm{e}^{-}$per carbon atom, from the naphthalene molecule to tetranitromethane. Since this species disappeared during a period of the order of about $1 \mathrm{~min}$, the shoulder near $285.8 \mathrm{eV}$ should be assigned to a

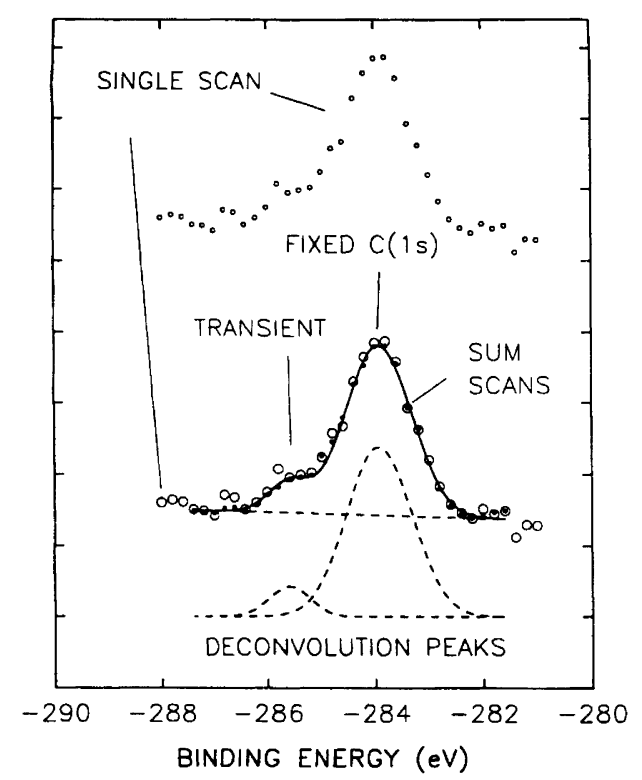

Fig. 4. A single narrow scan over the naphthalene portion of the $C(1 \mathrm{~s})$ spectrum is shown at the top. Seven such scans are added in the middle curve, and compared with the same single scan above. The transient shoulder is labelled. A deconvolution of the middle curve is shown at the bottom. transient species derived from naphthalene, either (naphthalene) ${ }^{\cdot+}$ of the (naphthalene) ${ }^{\cdot+}-\left(\mathrm{NO}_{2}\right)_{3} \mathrm{C}^{-}-\mathrm{NO}_{2}$ triad of stage III or the trinitromethylnaphthalenyl radical of stage IV of Fig. 1.

Solution kinetics measurements ${ }^{3}$ show that

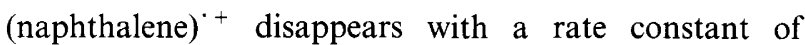
$2 \times 10^{11} \mathrm{dm}^{-3} \mathrm{~mol}^{-1} \mathrm{~s}^{-1}$ at room temperature following its initial formation via the triad, i.e., in a diffusion controlled reaction possessing almost zero activation energy, most likely by reaction with trinitromethanide ion. ${ }^{10}$ This means that the (naphthalene) ${ }^{+}$-trinitromethanide reaction must be very fast at $90 \mathrm{~K}$, thus leaving the neutral trinitromethylnaphthalenyl radical the most likely candidate for the transient species. Truly, the reaction between the latter and $\mathrm{NO}_{2}$ also must be a very fast step, as judged by the gas phase rate constant $\approx 2 \times 10^{10} \mathrm{dm}^{-3} \mathrm{~mol}^{-1} \mathrm{~s}^{-1}$ for reaction between $\mathrm{NO}_{2}$ and allyl radical at $300 \mathrm{~K},{ }^{11}$ presumably slower by a factor of 5-10 in the liquid phase, but such a value would yet leave the reaction IV (Fig. 1) as slower than III.

Calculations show that the strong electron-withdrawing effect of the trinitromethyl group on the ring carbons causes an average initial state shift of about $1 \mathrm{eV}$ compared with the experimental value of $1.8 \mathrm{eV}$. Here, because the molecule is a radical, final state effects, due to photoionization, as well as the proximity to the $\mathrm{NO}_{2}$ radical are believed to be important to better reproduce the experimentally measured shifts. In addition, the new species at $290 \mathrm{eV}$ is shifted by $2 \mathrm{eV}$ relative to the carbon of tetranitromethane, in good agreement with the calculated chemical shift for the trinitromethyl carbon of the trinitromethylnaphthalenyl radical.

When the transient shoulder at $285.8 \mathrm{eV}$ has disappeared, there remains a slightly broadened naphthalene $\mathrm{C}$ (1s) peak, and there exists a slight increase in intensity near $290 \mathrm{eV}$. These residual changes are assigned to the presence of stable nitro-trinitromethyl adducts to the naphthalene ring (stage $\mathrm{V}$ in Fig. 1). ${ }^{4}$ Calculations on the nitro-trinitromethyl adduct of Fig. 1 (stage V) again show that the carbon bonded to the three nitro groups is chemically shifted by $2.1 \mathrm{eV}$ relative to the peak of the carbon atom in TNM. This is in good agreement with experiment showing the appearance of the species at $290 \mathrm{eV}$ binding energy, corresponding to a shift of $2.0 \mathrm{eV}$. The calculated average chemical shift of the carbon atoms in the naphthalene ring of the adduct, excluding carbon directly bonded to the nitro and trinitromethyl groups is $1 \mathrm{eV}$ relative to the naphthalene peak. This explains the experimentally observed broadening of the naphthalene $C(1 \mathrm{~s})$ peak.

Finally, additional effects in the $\mathrm{C}(1 \mathrm{~s})$ spectrum are observed as the samples were warmed to $190 \mathrm{~K}$ the high binding energy peak at $292 \mathrm{eV}$ is shifted toward $288 \mathrm{eV}$, decreased in intensity and strongly broadened. This effect must largely be due to loss of volatile materials, residual TNM and possibly $\mathrm{N}_{2} \mathrm{O}_{4}$ formed by dimerization of $\mathrm{NO}_{2}$, leaving behind the adduct of stage $\mathrm{V}$ (Fig. 1). 


\section{Conclusions}

A light-induced, transient state, with a lifetime on the order of $1 \mathrm{~min}$, assigned to the trinitromethylnaphthalenyl radical resulting from recombination in the initially formed triad (naphthalene) ${ }^{\cdot+}-\left(\mathrm{NO}_{2}\right)_{3} \mathrm{C}^{-}-\mathrm{NO}_{2}$ may be identified in the C (1s) XPS spectra of condensed molecular layered samples consisting of tetranitromethane on naphthalene on gold at $90 \mathrm{~K}$. A steady state is eventually reached which consists of stable, chemically bonded species in the form of nitro-trinitromethyl adducts to naphthalene.

Acknowledgements. First of all we wish to express our thanks to Mikael Lögdlund, Piotr Barta and Carole Fauquet for help and fruitful discussions. Financial support for research on electron transfer processes (to L.E.) from the Swedish Natural Science Research Council (NFR) is gratefully acknowledged. Research on condensed molecular solids and polymers in Linköping is supported in general by grants from the Swedish Natural Science Research Council, the Swedish Research Council for Engineering Sciences (TFR), the Swedish National Board for Industrial and Technical Development (NUTEK), Hoechst Chemicals, Germany, and by the Commission of the European Community within the Training and Mobility of Researchers program (SELOA).

\section{References}

1. Kholmogorov, V. E. and Gorydskii, V. A. Russ. J. Phys. Chem. (Engl. Transl.) 46 (1972) 34.

2. Kochi, J. K. Acc. Chem. Res. 25 (1992) 39.

3. Bockmann, M. T. and Kochi, J. K. J. Chem. Soc., Perkin Trans. 2 (1994) 1901.

4. Eberson, L., Hartshorn, M. P. and Radner, F. Acta Chem. Scand. 48 (1994) 937.

5. Eberson, L., Hartshorn, M. P., Persson, O., Robinson, W. T. and Timmerman-Vaughan, D. J. Acta Chem. Scand. 49 (1995) 482.

6. Eberson, L., Radner, F. and Svensson, J. O. J. Chem. Soc., Chem. Commun. (1992) 1140.

7. Siegbahn, K., Nordling, C., Johansson, G., Hedman, J., Heden, P. F., Hamrin, V., Gelius, U., Bergmark, T., Werme, L. O., Manne, R. and Baer, Y. ESCA Applied to Free Molecules, North-Holland, Amsterdam 1971.

8. Riga, J., Pireaux, J. J., Caudano, R. and Verbist, J. J. Phys. Scr. 16 (1977) 346.

9. Clark, D. T. and Thomas, H. R. J. Polym. Sci., Polym. Chem. Ed. 15 (1977) 2843.

10. Eberson, L., Hartshorn, M. P., Radner, F. and Svensson, J. O. J. Chem. Soc., Perkin Trans 2 (1994) 1719.

11. Slagle, I. R., Yamada, F. and Gutman, D. J. Am. Chem. Soc: 103 (1981) 149.

Received April 8, 1997. 